\title{
COMO EU FALO COM VOCÊ? A COMUNICAÇÃO DO ENFERMEIRO COM O USUÁRIO SURDO
}

\section{HOW DO I TALK TO YOU? THE COMMUNICATION OF THE NURSE WITH THE DEAF USER}

\section{¿CÓMO HABLO CON USTED? LA COMUNICACIÓN DEL ENFERMERO CON EL USUARIO SURDO}

\author{
Imaculada Pereira Soares ${ }^{1}$ \\ Elis Mayara Messias de Lima $^{2}$ \\ Ana Caroline Melo dos Santos ${ }^{3}$ \\ Cíntia Bastos Ferreira ${ }^{4}$
}

Como citar este artigo: Soares IP, Lima EMM, Santos ACM, Ferreira CB. Como eu falo com você? a comunicação do enfermeiro com o usuário surdo. Rev baiana enferm. 2018;32:e25978.

\begin{abstract}
Objetivo: descrever os saberes e as práticas de profissionais enfermeiros da atenção básica na assistência do usuário surdo. Metodologia: estudo exploratório descritivo, com abordagem qualitativa. A coleta de dados foi realizada por meio de entrevista semiestruturada aplicada aos enfermeiros que atuavam nas unidades básicas de saúde do município de Arapiraca, Alagoas, Brasil. O material foi submetido à técnica de análise de conteúdo de Bardin. Resultados: emergiram das falas dos sujeitos as unidades temáticas: "Desconhecimento sobre a Língua Brasileira de Sinais" e "Práticas utilizadas pelos enfermeiros para viabilizar a interação com usuários surdos". Conclusão: os sujeitos do estudo não sabiam comunicar-se por meio da Língua Brasileira de Sinais, consideravam a ausência de acompanhante como barreira para a assistência aos usuários surdos e precisavam de outros meios para se comunicar com esses usuários, a exemplo da escrita, com os usuários alfabetizados, e a utilização de gestos ou leitura labial.
\end{abstract}

Descritores: Comunicação. Enfermagem. Surdez.

Objective: to describe the knowledge and practices of primary health care nurses relating to the care of the deaf user. Methodology: descriptive exploratory study, with a qualitative approach. Data collection was performed through a semi-structured interview applied to nurses who worked in the primary bealth care units in the city of Arapiraca, Alagoas, Brazil. The material was submitted to the Bardin content analysis technique. Results: the thematic units emerged from the speeches of the subjects: "Unawareness of the Brazilian Sign Language" and "Practices used by nurses to enable interaction with deaf users". Conclusion: the study subjects did not know how to communicate using the Brazilian Sign Language and considered the absence of a chaperone as a barrier to attending deaf users and needed other means to communicate with these users, such as writing, with literate users, and the use of gestures or lip reading.

Descriptors: Communication. Nursing. Deafness.

Objetivo: describir saberes y prácticas de profesionales enfermeros de la atención básica en la atención al usuario sordo. Metodología: estudio exploratorio descriptivo, con abordaje cualitativo. Recolección de datos realizada por

\footnotetext{
Enfermeira. Mestranda em Enfermagem pela Universidade Federal de Alagoas. Maceió, Alagoas, Brasil. imaculada.enfermagem@gmail.com

Enfermeira. Especialista em Enfermagem do Trabalho. Maceió, Alagoas, Brasil.

Enfermeira. Doutoranda em Ciência da Saúde pela Universidade Federal de Alagoas. Maceió, Alagoas, Brasil.

Enfermeira. Doutoranda em Educação pela Universidade Federal de Alagoas. Professora da Universidade Federal de Alagoas. Arapiraca, Alagoas, Brasil.
} 
medio de entrevista semiestructurada aplicada a enfermeros que actuaban en las unidades básicas de salud del municipio de Arapiraca, Alagoas, Brasil. Material sometido a la técnica de análisis de contenido de Bardin. Resultados: emergieron de las hablas de los sujetos las unidades temáticas: "Desconocimiento sobre la Lengua Brasileña de Señales" y "Prácticas utilizadas por enfermeros para viabilizar la interacción con usuarios sordos". Conclusión: los sujetos del estudio no sabian comunicarse por medio de la Lengua Brasileña de Señales, consideraban la ausencia de acompañante como barrera para atención a los usuarios sordos y necesitaban otros medios para comunicarse con esos, a ejemplo de la escritura, con usuarios alfabetizados, y utilización de gestos o lectura labial.

Descriptores: Comunicación. Enfermería. Sordera.

\section{Introdução}

A surdez é compreendida atualmente como uma especificidade de pessoa que se diferencia de outros seres em razão da sua forma de comunicação. Esforços lançados por resistências surdas possibilitaram o fortalecimento de um "novo" grupo social, no qual o surdo constitui-se como um ser que se diferencia dos demais por utilizar uma linguagem gesto-visual, não devendo ser qualificado como deficiente ou visto como doente ${ }^{(1)}$.

Em uma sociedade em que a língua oral é prevalente e, portanto, os indivíduos devem adequar-se a ela para se integrarem no meio social, a população não está preparada para acolher o surdo. O mesmo acontece no encontro entre um ser surdo e o profissional de saúde. $\mathrm{Na}$ maior parte dos casos, essa interlocução se dá por meio da linguagem verbal, seja na sua forma oral (tentando fazer com que o usuário surdo consiga ler os lábios ou com a dependência de um acompanhante tradutor), seja na sua forma escrita, o que cria obstáculos na comunicação. Esta pode também tentar efetivar-se pelo uso de gestos. Já a Língua Brasileira de Sinais (Libras), oficial da comunidade surda no Brasil, é pouco utilizada pelos profissionais de saúde ${ }^{(2)}$.

Tendo em vista essa realidade e considerando que o pilar para a atenção em saúde (desde a anamnese até o momento das orientações) é a boa comunicação entre o profissional e o usuário, é de se esperar que, no momento em que isso se torna falho, são grandes as possibilidades de equívocos de diagnósticos e, consequentemente, de problemas em sua solução. A ausência de qualificação dos profissionais de saúde pode criar prejuízo durante a assistência, resultando em constrangimento, diagnóstico errôneo, dificuldade de elaborar corretamente o prontuário e tratamento inadequado para a possível patologia ${ }^{(3)}$. Ademais, o acolhimento nos serviços de saúde é necessário para que o direito à saúde seja garantido. Para o usuário surdo, a barreira de comunicação mostra-se como uma dificuldade que tem como consequência o desrespeito aos seus direitos ${ }^{(4)}$.

Em se tratando de disparidade, a prevalência de depressão entre usuários com problemas na audição mostrou-se maior em um estudo realizado nos Estados Unidos ${ }^{(5)}$. Essa desigualdade implica também no conhecimento sobre saúde, já que adolescentes surdos demonstraram um nível de alfabetização em saúde menor, quando comparados a adolescentes ouvintes ${ }^{(6)}$.

Os profissionais de enfermagem têm uma responsabilidade legal e ética de proporcionar cuidados de saúde para usuários surdos que usam a linguagem de sinais, da mesma forma que os fornecem a outros usuários, com comunicação efetiva, autonomia e confidencialidade. Todavia, esta não tem sido a realidade ${ }^{(7)}$.

Tendo em vista que a comunicação é um fator-chave na interação dos usuários com o sistema de saúde, essa interação com usuários surdos fica inicialmente comprometida pela barreira de comunicação que se estabelece. Portanto, em uma sociedade que é principalmente ouvinte, é de se esperar que haja um impacto negativo na saúde e comprometimento de alguns direitos individuais e coletivos ${ }^{(8)}$. A fim de contribuir para o conhecimento científico na 
área da enfermagem, colaborando também para suprir lacunas existentes acerca da temática proposta, este estudo tem como questão norteadora: Quais os saberes e as práticas de enfermeiros da atenção básica, frente a assistência ao usuário surdo?

O estudo teve o objetivo de descrever os saberes e as práticas de profissionais enfermeiros da atenção básica, na assistência do usuário surdo.

\section{Método}

Trata-se de um estudo exploratório descritivo, com abordagem qualitativa. O cenário de estudo foram Unidades Básicas de Saúde da Estratégia Saúde da Família do município de Arapiraca, Alagoas, Brasil.

O Brasil tem 23,91\% de usuários com alguma deficiência e, destes, 26,6\% estão no Nordeste. No estado de Alagoas, o percentual de usuários com alguma deficiência é maior do que o percentual brasileiro, chegando a 27,5\%. Aproximadamente $6 \%$ da população alagoana apresenta alguma deficiência auditiva. Arapiraca corresponde à segunda maior cidade do estado em número de pessoas e em importância social e econômica, ficando atrás apenas da capital Maceió. O município pesquisado possui 234.185 habitantes $^{(9)}$, com 67 equipes da Estratégia Saúde da Família com 219 enfermeiros.

Foram entrevistados 20 enfermeiros atuantes nas Unidades Básicas de Saúde cenário desta pesquisa. O número de entrevistados seguiu o critério de saturação dos dados, compreendido como o momento em que os dados começam a se repetir em um determinado número de entrevistas.

O critério de inclusão foi ser enfermeiro que atuasse na Estratégia Saúde da Família. O único critério de exclusão foi estar afastado das funções por qualquer motivo na época da coleta de dados.

Os instrumentos de coleta de dados utilizados foram o questionário, para caracterizar o perfil social dos enfermeiros, e a entrevista com um roteiro de perguntas abertas. As entrevistas foram realizadas individualmente, no período de maio a julho de 2015. As entrevistas foram gravadas em um aparelho celular e depois transcritas, para posterior análise. Os profissionais convidados a participar foram orientados quanto aos objetivos da pesquisa e aqueles que aceitaram, assinaram o Termo de Consentimento Livre Esclarecido (TCLE). Para garantir o anonimato dos sujeitos participantes da pesquisa, foi utilizada a letra inicial da palavra enfermeiro (E) seguida de números sequenciais (E1, E2, E3...).

Para análise dos dados, foi utilizada a técnica de análise de conteúdo proposta por Bardin ${ }^{(10)}$. Na fase de pré-análise, os textos transcritos das entrevistas foram organizados para possibilitar a sistematização das ideias. Esta fase constituiu-se de leitura flutuante, para conhecimento do texto, e demarcação do que seria analisado. Na segunda fase, o material foi explorado para fins de definição das categorias de análise. Por fim, os resultados foram tratados, para permitir inferências e interpretações feitas pelos pesquisadores.

$O$ projeto de pesquisa obedeceu às normas que regem pesquisas com seres humanos - Declaração de Helsinque (1964) e Resolução n. 466/12 do Ministério da Saúde - e foi encaminhado ao Comitê de Ética em Pesquisa da Universidade Federal de Alagoas (UFAL), tendo sido aprovado sob o protocolo número 1.026.849.

\section{Resultados}

A faixa etária dos enfermeiros participantes desta pesquisa variou entre 26 a 65 anos, sendo apenas dois sujeitos com idade maior que 40 anos. Quanto ao sexo, 17 eram mulheres e 3 eram homens. No que se refere ao tempo de experiência na profissão, houve uma variação entre 1 a 30 anos, sendo mais de 50\% com 5 anos ou mais. Quanto ao tempo de atuação na Estratégia Saúde da Família, a variação ficou entre 1 a 18 anos, tendo, a maior parte, menos de 5 anos de atuação.

Duas Unidades Temáticas emergiram dos depoimentos e são apresentadas na sequência: "Desconhecimento sobre a Língua Brasileira de 
Sinais" e "Práticas utilizadas pelos enfermeiros para viabilizar a interação com usuários surdos”.

\section{Desconbecimento sobre a Lingua Brasileira de Sinais}

Com base nos depoimentos dos sujeitos da pesquisa, chama a atenção o fato de todos os 20 profissionais entrevistados relatarem não saber a Língua Brasileira de Sinais (Libras). Alguns disseram que já tiveram contato superficial, mas nenhum a dominava.

Não sei Libras. (E1).

Não, eu não sei [Libras]. Nunca fui preparada, nunca fiz nenbum treinamento para esse sentido. (E8).

Por não ter o curso de Libras, não saber a linguagem dos sinais, isso dificulta no entendimento e fica na base do achismo, não é? (E11).

É importante mencionar que todos os 20 entrevistados afirmaram ter prestado assistência a usuários com deficiência auditiva durante sua vida profissional. E por não saberem Libras, demonstraram, em suas falas, dificuldade de comunicação com o usuário surdo. Um dos entrevistados, inclusive, disse que nem presta assistência, caso o usuário venha sozinho.

Lembro que, uma consulta, ela veio sozinha, mas foi bem complicada, assim, a comunicação. (E6).

Se ela tivesse vindo sozinha, o atendimento não seria satisfatório. (E18).

Eu não atendo [...] se não vier acompanhado. (E7).

Vê-se, nesta Unidade Temática, que o fato de não saber a Libras é apontado como um problema na relação entre o profissional de saúde e o portador de surdez.

\section{Práticas utilizadas pelos enfermeiros para viabilizar a interação com usuários surdos}

O enfermeiro precisa desenvolver formas de interação com os usuários, para completar a atenção em saúde. Os depoentes relataram as seguintes práticas utilizadas para viabilizar a comunicação: presença de um acompanhante durante as consultas; utilização da escrita; uso de linguagem corporal.
Quase todos os enfermeiros relataram que o usuário que vem para o atendimento na unidade com acompanhante que é do seu convívio, na maioria dos casos sabe Libras e conhece suas necessidades e dúvidas, há possibilidade de sucesso na transmissão de informações.

Acho que facilita, para o atendimento da gente, o acompanbante que sabe falar. (E7).

Já fiz pré-natal com uma pessoa que tinha problema auditivo, era surda-muda, e aí vinha sempre a irmã dela, ou a mãe, ou o esposo na consulta e aí a gente acabava fazendo essa comunicação através do acompanhante. (E6).

Um problema que pode ser apontado nessas situações e que os sujeitos do estudo não consideraram em suas falas, é que a presença de uma terceira pessoa na assistência pode quebrar a relação de confiança entre profissional e usuário e, com certeza, é um impedimento à privacidade e até ao sigilo.

A utilização da escrita foi relatada por muitos entrevistados como um fator que auxiliava bastante no contato com usuários surdos, mas, para tanto, era preciso garantir que o usuário soubesse ler e escrever.

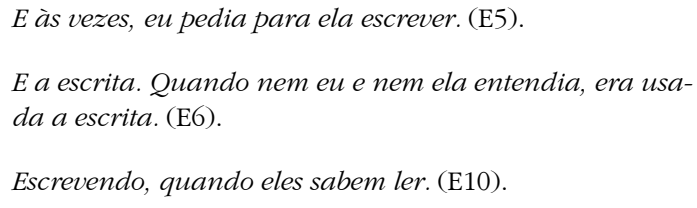

A linguagem corporal e a utilização de outros sentidos, como os gestos e a leitura labial, foram também consideradas facilitadoras da comunicação.

\footnotetext{
Foi uma mistura de gestos, leitura labial. (E20).

Considero como melhor estratégia utilizada para este atendimento a chamada leitura labial. Comecei a perceber, nas consultas subsequentes, que, quando eu falava lentamente e olbo no olho dela, ela expressava um contentamento e um entendimento melhor. (E15).
}

Depreende-se, nesta Unidade Temática, que os profissionais sujeitos desta pesquisa precisaram usar diversas formas de comunicação para viabilizar algum tipo de interação, já que nenhum deles sabia Libras e todos já prestaram assistência a algum usuário com problema auditivo durante sua vida profissional. 


\section{Discussão}

O desconhecimento da Libras é relatado nesta pesquisa como a principal dificuldade que o profissional enfermeiro enfrenta quando precisa assistir um usuário surdo. Uma pesquisa realizada em Porto Alegre (RS) concluiu algo semelhante, ao afirmar que o principal desafio para os profissionais de saúde que cuidam de usuários surdos é conseguir estabelecer uma possibilidade de interação por meio da substituição da linguagem verbal à qual estão habituados, para a linguagem de sinais ${ }^{(4)}$.

Outro estudo, que buscou discutir a importância e eficácia das consultas de enfermagem para os usuários surdos, diz que o desconhecimento de Libras, por parte dos profissionais de saúde, deixa-os insatisfeitos e acarreta angústia. Normalmente não conseguem fazer-se entender e também não conseguem compreender as orientações recebidas. Para o mesmo estudo, quando os profissionais de saúde sabem Libras e conseguem comunicar-se de forma eficaz com seus usuários, há uma assistência mais humanizada e respeitosa, possibilitando o comportamento inclusivo ${ }^{(11)}$.

$\mathrm{Na}$ presente pesquisa, pelo fato de os profissionais entrevistados não saberem Libras, a ausência de um acompanhante no momento da consulta foi apontada como barreira de comunicação. O mesmo foi detectado em pesquisa realizada nos Estados Unidos da América, na qual os enfermeiros preferiam ter um acompanhante atuando como intérprete, para facilitar a comunicação ${ }^{(7)}$.

Cursos sobre a Libras para profissionais da saúde surgem, então, como melhor opção para uma comunicação eficaz com os usuários surdos ou mesmo com seus parentes. Uma alternativa, por exemplo, são os cursos a distância ${ }^{(12)}$. Quanto mais profissionais consigam aprender Libras, maior a possibilidade de respeito à inclusão social e à cultura do surdo ${ }^{(7)}$.

Por outo lado, tendo em vista que os EUA é um país desenvolvido, essa situação sugere que a deficiência de saberes com relação à Libras é uma dificuldade que requer mais do que melhores condições econômicas do país. O desenvolvimento de programas de treinamento efetivos e acessíveis para os profissionais que trabalham com usuários surdos e com deficiência auditiva, por exemplo, exigirá um esforço colaborativo entre agências de resposta a emergências, organizações de saúde pública e membros das comunidades afetadas ${ }^{(13)}$. Não se pode deixar de levar em consideração que, em detrimento da forma como a surdez foi associada à esfera patológica, criou-se um estigma que colocou os indivíduos portadores dessa condição, por vezes, em um local de subalternidade marcado pelo preconceito e pela exclusão social ${ }^{(14)}$.

Outra pesquisa, que também traz resultados semelhantes, relata que, além do fato de não conhecerem Libras, existem problemas que o enfermeiro pode apontar como elementos que dificultam a comunicação, e que podem estar ligados, por exemplo, à falta de um acompanhante que atue como intérprete e até à deficiência de escolaridade do surdo ou do acompanhante. Entretanto, segundo a pesquisa citada, mesmo que esses problemas sejam sanados, não há garantia de comunicação efetiva entre o profissional e o usuário, pois tal comunicação só acontece quando são incorporados a cultura e os sentidos da interação ${ }^{(11)}$. Ademais, o uso de uma pessoa da família como tradutor pode restringir as ações em saúde, pois esse parente pode não compreender as orientações ou não interpretar para o usuário de forma clara ${ }^{(15)}$.

Nesta pesquisa, como os enfermeiros não sabiam Libras, eles apontaram estratégias que escolhiam utilizar para conseguir alguma interação com os usuários surdos, tais como: presença de um acompanhante nas consultas e utilização da escrita e da linguagem corporal.

As ações e estratégias para a assistência aos usuários surdos deveriam ser de âmbito geral, orientadas por políticas públicas e planejadas pela gestão junto com os serviços de saúde. Entretanto, normalmente, as ferramentas utilizadas com o intuito de viabilizar a comunicação são parte de iniciativas individuais e pontuais. Cada um vai arrumando estratégias, à medida que surgem as necessidades. Há, portanto, uma 
fragmentação das ações que não possibilita a aquisição de novas práticas sólidas para promover a acessibilidade do portador de surdez ${ }^{(16)}$.

Em uma pesquisa que objetivou chamar a atenção dos profissionais de saúde e docentes, os entrevistados alegaram que, mesmo que o profissional de saúde não domine a Língua Brasileira de Sinais, ele pode estabelecer alguma comunicação com seus usuários surdos, utilizando outros meios, como os gestos, a escrita e a fala articulada, para a leitura labial ${ }^{(12)}$.

O profissional enfermeiro está sujeito a muitas dificuldades com relação à compreensão da mensagem transmitida pelos deficientes auditivos, assim como para passar informações e orientações para eles. Por isso, utiliza diversas formas de comunicação, como mímica ou linguagem escrita, que, entretanto, não são eficientes e não garantem o tratamento correto. As necessidades de comunicação vão além da interpretação de gestos e notas e pensar que seja suficiente demonstra falta de consciência das reais necessidades de comunicação ${ }^{(7)}$. Deste modo, é necessário que os enfermeiros dominem a Língua Brasileira de Sinais $^{(11)}$.

Em relação às limitações do estudo, existem algumas questões que precisam ser consideradas. Primeiro, os sujeitos do estudo foram profissionais apenas da Atenção Básica de Saúde e a problemática da dificuldade de assistência ao usuário surdo deve estender-se também para a atenção secundária e terciária. Além disso, foram selecionados apenas profissionais de enfermagem de nível superior, quando poderia ter sido considerada toda a equipe de enfermagem ou, melhor ainda, toda a equipe de saúde.

\section{Conclusão}

Conclui-se que os sujeitos do estudo não sabiam comunicar-se por meio da Língua Brasileira de Sinais, consideravam a ausência de acompanhante como barreira para a assistência aos usuários surdos e precisavam de outros meios para se comunicar com esses usuários, a exemplo da escrita, com os usuários alfabetizados, e a utilização de gestos ou leitura labial.
Entretanto, esses meios alternativos nem sempre são viáveis, pois, ainda assim, existem inúmeras barreiras que dificultam todo o processo de comunicação entre as partes.

Os enfermeiros reconheceram a necessidade de se comunicar com mais qualidade com usuários surdos e entenderam que a interação entre profissional e usuário era fundamental para garantir sucesso na assistência à saúde. Há uma parcela da população com surdez que precisa ter os seus direitos de saúde assegurados, assim como qualquer outro usuário. Por isso, os profissionais dessa área precisam estar aptos a acolher seus usuários. Contudo, para conseguirem prestar uma atenção de qualidade, precisam entender que a efetividade da comunicação é uma prioridade.

Nesta pesquisa, todos os enfermeiros entrevistados já tiveram a experiência de prestar assistência a pelo menos um usuário surdo, situação que se repete diversas vezes, em qualquer lugar. Portanto, o serviço de saúde e o profissional precisam estar preparados para receber esses usuários.

Uma questão surgida diante da necessidade de capacitação profissional é refletir sobre os currículos dos cursos de formação para a saúde e a possibilidade de incluir a disciplina de Libras como componente obrigatório e garantir a qualidade da comunicação. Este trabalho também traz à discussão a ideia de que os gestores da saúde devem entender essa demanda e providenciar capacitações para os trabalhadores dessa área que já estão no serviço e podem ter contato direto com o público especificado.

Nesta pesquisa, foi possível traçar um panorama dos saberes dos enfermeiros acerca de Libras e reconhecer as práticas que adotam para se comunicar com os usuários surdos. Perceber que há uma deficiência de conhecimento sobre a Língua Brasileira de Sinais é útil para pensar em atividades de educação permanente, para que a assistência à saúde assuma uma postura de respeito e inclusão. Também é importante planejar ações que possibilitem a comunicação, mas que também estabeleçam uma relação de 
confiança e garantam preceitos, como o sigilo e a privacidade.

É preciso que novos estudos sobre a temática sejam realizados com outros profissionais da equipe de saúde, além dos enfermeiros, e incorporem os demais níveis de atenção à saúde.

\section{Colaborações:}

1. concepção, projeto, análise e interpretação dos dados: Imaculada Pereira Soares, Elis Mayara Messias de Lima, Ana Caroline Melo dos Santos e Cintia Bastos Ferreira;

2. redação do artigo e revisão crítica relevante do conteúdo intelectual: Imaculada Pereira Soares, Elis Mayara Messias de Lima, Ana Caroline Melo dos Santos e Cíntia Bastos Ferreira;

3. aprovação final da versão a ser publicada: Imaculada Pereira Soares, Elis Mayara Messias de Lima, Ana Caroline Melo dos Santos e Cíntia Bastos Ferreira.

\section{Referências}

1. Lins HAM, Nascimento LCR. Algumas tendências e perspectivas em artigos publicados de 2009 a 2014 sobre surdez e educação de surdos. Pro-posições. 2015;26(3):27-40.

2. Oliveira YCA, Celino SDM, Costa GMC. Comunicação como ferramenta essencial para assistência à saúde dos surdos. Physis (Rio J). 2015;25(1):307-20.

3. Gomes LF, Machado FC, Lopes MM, Oliveira RS, Holanda BM, Silva LB, et al. Conhecimento de Libras pelos médicos do Distrito Federal e atendimento ao paciente surdo. Rev bras educ méd. 2017;41(4):551-6.

4. Tedesco JR, Junges JR. Desafios da prática do acolhimento de surdos na atenção primária. Cad Saúde Pública. 2013;29(8):1685-9.

5. Li CM, Zhang X, Hoffman HJ, Cotch MF, Themann CL, Wilson MR, et al. Hearing impairment associated with depression in US adults, National Health and Nutrition Examination Survey 2005-2010. JAMA Otolaryngol Head Neck Surg [Internet]. 2015 [cited 2018 Jun 10];140(4):293-302. Available from: http:// www.ncbi.nlm.nih.gov/pubmed/24604103
6. Smitha SR, Samar VJ. Dimensions of Deaf/Hardof-Hearing and Hearing Adolescents' Health Literacy and Health Knowledge. J Health Commun. 2016;21(sup2):141-54.

7. Pendergrass KM, Nemeth L, Newman SD, Jenkins $\mathrm{CM}$, Jones EG. Nurse practitioner perceptions of barriers and facilitators in providing health care for deaf American Sign Language users: a qualitative socio-ecological approach. J Am Assoc Nurse Pract. 2017 Jun;29(6):316-23.

8. Rodríguez-Martín D, Rodríguez-García C, Falcó-Pequeroles A. Ethnographic analysis of communication and the deaf community's rights in the clinical context. Contemp Nurse [Internet]. 2018 Mar [cited 2018 Jun 10];6178(1):144-73. Available from: DOI: 10.1080/10376178.2018.1441731

9. Instituto Brasileiro de Geografia e Estatística. Censo demográfico: resultados preliminares da amostra. Rio de Janeiro; 2017 [Internet]. [cited 2018 Jun 10]. Available from: https://ww2.ibge.gov.br/home/ estatistica/populacao/censo2010/default.shtm

10. Bastos MHR, Oliveira UR. Análise de Discurso e Análise de Conteúdo: um breve levantamento bibliométrico de suas aplicações nas ciências sociais aplicadas da Administração. Trabalho apresentado no $12^{\circ}$ Simpósio de Excelência em Educação e Tecnologia; 2015 out 28-30. Resende; 2015 [cited 2018 Jun 11]. Available from: https:// www.aedb.br/seget/arquivos/artigos15/26322295. pdf

11. Trecossi MO, Ortigara EPF. Importância e eficácia das consultas de enfermagem ao paciente surdo. Rev enferm. 2013;9(9):60-9.

12. García ER, García GR, Oliva MA, Duquesnes MQ, Francia YH. Curso virtual sobre la lengua de señas cubana para los estudiantes de Medicina. Edumecentro. 2017;9(2):93-109.

13. Kamau PW, Ivey SL, Griese SE, Qari SH. Preparedness training programs for working with deaf and hard of hearing communities and older adults: lessons learned from key informants and literature assessments. Disaster Med Public Heal Prep. 2017 Oct;11:1-9.

14. Abreu FSD, Silva DNH, Zuchiwschi J. Surdos e homossexuais: a (des)coberta de trajetórias silenciadas. Temas psicol (Internet). 2015 [cited 2018 Jun 10];23(3):607-20. Available from: DOI: 10.9788/TP2015.3-07 
15. Miranda RS, Shubert CO, Machado WCA. A comunicação com pessoas com deficiência auditiva: uma revisão integrativa. J Res Fundam Care. 2014;6(4):1695-1706.

16. Sales AS, Oliveira RF, Araújo EM. Inclusão da pessoa com deficiência em um Centro de
Referência em DST/AIDS de um município baiano. Rev bras enferm. 2013;66(2):208-14

Recebido: 20 de março de 2018

Aprovado: 26 de junho de 2018

Publicado: 3 de setembro de 2018

A Revista Baiana de Enfermagem utiliza a Licença Creative Commons - Atribuição-NãoComercial 4.0 Internacional.

https://creativecommons.org/licenses/by-nc/4.0/

Este artigo é de acesso aberto distribuído sob os termos da Licença Creative Commons (CC BY-NC).

Esta licença permite que outros remixem, adaptem e criem a partir do seu trabalho para fins não comerciais.

Embora os novos trabalhos tenham de lhe atribuir o devido crédito e não possam ser usados para fins comerciais, os usuários não têm de licenciar esses trabalhos derivados sob os mesmos termos. 